\title{
Comparison between various strategies for the disease-gene mapping using linkage disequilibrium analyses: studies on adenine phosphoribosyltransferase deficiency used as an example
}

Received: 24 March 2004/ Accepted: 19 May 2004 / Published online: 28 July 2004

(C) The Japan Society of Human Genetics and Springer-Verlag 2004

\begin{abstract}
Recently, linkage disequilibrium analyses have been used to detect disease-causing loci based on the common disease-common variant hypothesis. To see what methods can effectively identify the genes, we have to apply them to the practical data obtained from the human population. We extensively performed linkage disequilibrium and haplotype analyses on adenine phosphoribosyltransferase $(A P R T)$ genes in both control and deficient subjects. To examine the power to detect disease-causing loci, we analyzed SNPs, STRPs, and VNTR within and around the APRT gene. When only SNPs were used, $P$ values did not necessarily show significant difference, even at loci close to the mutation site for $A P R T^{*} J$ that is exclusively observed among Japanese. However, the examination of the same samples with haplotypes based on the haplotype block data gave sufficient significance. In the case of STRP and VNTR, some single-marker loci showed significant difference. Our study suggested that the use of haplotype analysis based on the haplotype-block structure is more
\end{abstract}

S. Kuno $\cdot$ A. Taniguchi $\cdot$ A. Saito $\cdot$ S. Tsuchida-Otsuka

N. Kamatani

Institute of Rheumatology,

Tokyo Women's Medical University, Tokyo, Japan

S. Kuno $(\square)$

Laboratory for Clinical Genome Informatics,

Translational Research Informatics Center,

Foundation for Biomedical Research and Innovation,

1-5-4 Minatojima-minamimachi, Chuo-ku,

Kobe 650-0047, Japan

E-mail: omoikane@tri-kobe.org

Tel.: +81-78-3032760

Fax: $+81-78-3032761$

A. Saito

Advanced Research Laboratory, Hitachi Ltd.,

Tokyo, Japan

N. Kamatani

Division of Genomic Medicine,

Department of Advanced Biomedical Engineering and Science,

Tokyo Women's Medical University,

Tokyo, Japan powerful than single-marker locus analysis for the detection of disease-related loci.

Keywords Adenine phosphoribosyltransferase deficiency $\cdot$ Linkage disequilibrium $\cdot$ Case-control analysis $\cdot$ SNP $\cdot$ Microsatellite $\cdot$ VNTR $\cdot$ Haplotype

\section{Introduction}

Common disease-common variant hypothesis predicts that causative mutations responsible for a common disease are likely to be attributed to common variants. The common variants presumably derived from common ancestor mutations. In such a case, the causative mutations can be identified by searching for the polymorphisms that are not the real causes of the disease but are in linkage disequilibrium (LD) with the causative mutations.

Recent genomic studies have been focused on the selection of single nucleotide polymorphisms (SNPs), short tandem repeat polymorphisms (STRPs; microsatellites), and variable numbers of tandem repeat (VNTR) that are in LD with common diseases such as diabetes mellitus (Mohlke et al. 2001) and hypertension (Angius et al. 2002). Such a selection has been attempted after narrowing the candidate regions by the linkage analyses or by directly searching the entire chromosomes. However, very few such attempts have so far been successful.

In the present study, we have tested the hypothesis as to whether attempts to identify the disease mutations can be successful by looking for polymorphisms that are in LD with the disease mutation. If it can be successful, we intended to ask what strategies would be optimal to solve this problem. Some researchers claimed that SNPs are superior to STRPs for that purpose while others argued against that. We also intended to test whether the use of haplotypes in addition to SNPs and STRPs is useful for finding the disease locus. 
To do this, we selected a genetic disease that is rather common and whose disease mutations have already been identified. We concealed the causative mutations in the samples and examined whether the search for the disease mutations by the method of LD in a case-control study would be successful and, if so, what strategies would be optimal. Adenine phosphoribosyltransferase (APRT) deficiency is an autosomal recessive genetic disease (Kelley et al. 1968; Henderson et al. 1969; Cartier et al. 1974) that is common, as a genetic disease, among Japanese, although it is far less common than common diseases such as diabetes, hypertension, rheumatoid arthritis, and chronic glomerulonephritis. This $A P R T$ deficiency includes partial deficiency called Type II $\left(A P R T^{*} J\right)$, which is observed only in Japanese (Kamatani et al. 1985), and complete deficiency called as Type I $\left(A P R T^{*} Q 0\right)$, which is observed in other ethnic groups as well (Sahota et al. 1995). The incidence of the defective Type II gene has been estimated to be $3.65 \times 10^{-3}$ (Kamatani et al. 1996), and the incidence of the homozygotes has been estimated to be $1.33 \times 10^{-5}$. Previous studies have shown that the majority of disease chromosomes among the Japanese had a few common variants (Kamatani et al. 1992). By analysis of a few franking RFLPs, it was suggested that the majority of variants derived from a few common ancestors (Kamatani et al. 1990).

$A P R T$ is coded for by a gene $2.6 \mathrm{kbp}$ in length and located in 16q24.3 (Fratini et al. 1986). The defective mutations of this gene cause an autosomal recessive disease APRT deficiency. The clinical features of this disease include 2,8-dihydroxyadenine crystalluria (Laxdal and Jonasson 1988) and urolithiasis (Debray et al. 1976). The penetrance of this disease was estimated to be $85 \%$ in Type I homozygotes (Sahota et al. 1995), and the severity of the disease fluctuates very much from without symptoms to acute renal failure leading to hemodialysis or renal implantation. Such a fluctuation probably depends on environmental factors.

The analysis of mutations for APRT deficiency among Japanese patients has elucidated that about $78 \%$ of the defective chromosomes contain a single mutation designated $A P R T^{*} J$ (Kamatani et al. 1989). Including the most frequent mutation, three different changes, $A P R T^{*} J$ (Hidaka et al. 1988), $A P R T^{*} Q 0 c$ (Mimori et al. 1991), and $A P R T^{*} Q 04$ (Kamatani et al. 1992), in the nucleotide sequence have been found to account for $96 \%$ of all the defective mutations (Kamatani et al. 1992). The above characteristics in the region of the defective gene are what we expected to find in the responsible genes for the diseases compatible with the common disease-common variant hypothesis. Therefore, the precise analyses of LD around the APRT gene on defective and control chromosomes may give us valuable information as to how we can find the responsible mutations using the LD.

\section{Subjects and methods}

Subjects DNA samples were obtained from 174 Japanese including $42 A P R T^{*} J$ homogenous subjects, 14 $A P R T^{*} \mathrm{~J} / Q O$ heterogeneous subjects (containing $9 \mathrm{~J} /$ $Q 0 c, 2 \mathrm{~J} / Q 04$ and $3 \mathrm{~J} / Q 0 \mathrm{u}), 24 A P R T^{*} Q 0$ homogenous subjects (containing $13 \mathrm{QOc} / \mathrm{QOc}, 3 \mathrm{Q04} / \mathrm{Q} 04,2 \mathrm{QOs} / \mathrm{QOs}$, $5 Q 0 u / Q O u$ and $1 Q 0 c / Q 0 u$, and 94 control subjects.

Laboratory analysis PCR was performed in $50 \mu \mathrm{l}$ reaction mixture containing $200 \mu \mathrm{M}$ deoxyribonucleoside triphosphates (dNTPs), $1 \mathrm{nM}$ PCR forward and reverse primers, $2.5 \mathrm{U}$ AmpliTaq Gold (Applied Biosystems), 100 ng DNA sample, and PCR buffer (Applied Biosystems). The forward and reverse PCR primer sequences for the products including marker locus are No. 1 (GCAATGCTCCGCGAGGTAT and GATAGAATGCGGCTAACCCA), No. 2 (TGGCAGGCTATGGTTCCC and GGCTTCTCCTCTGTTGAGCA), No. 3 (GGGCCTTGGTGGTTCTC and CTTCTGTGGTAGCGGTCCT), No. 4 (AGGCATCCTGACCAGAGTCT and CAAGTGCCCTATGACAG), No. 5 (ACTGCCCAGGAGCTGAGGAT and GACTGCGGTGGTCCCAT), No. 6 (GGGTCGCCACGCAGAG and GAGGCAGGAGAATCGCTTGAA), No. 7 (ACCGCCCGCAGCCAGAGACC and mismatch primer GCTGGACAAGGCCGCGGAGC), No. 8 (CTTGTCCTGCCCAGCTTCTC and GGCGAACATGGTGAAACCC), No. 9, No. 10, No. 11, and No. 12 (AGAGGGTGGTCGTCGTGGAT and GAACAGGAGGACAGGAGACG), No. 13, No. 14, No. 15, and No. 16 (CTGCACTCTGACCTGGAAGC and GGGAGACCCTTACCACCAGT), No. 17 and No. 18 (GGAGCCACAACACTGCCAGA and CTTCCCGTACTCCAGGGAAT), No. 19 (CCCACCCCAGGCGTGGTATT and GCAGCTCTGCACCAGGGCTT), No. 20 (CATCTCGCCCGTCCTGAA and CCTGGGTGGGCATTCCGTGA), No. 21 (GAGGCTGAGGCGGGAGAATG and GAGGGAGGCCGGAAGGTGT), No. 22 (CTGGACGTGGACGGCTGAAGTGTAG and TTACAATTCAGCTTTGGCCTGGCAGTTACT), No. 23 (GTGAAACGCCACACGCACAG and CCCCACGAGCAGCTTCCCTC), No. 24 (CACCATGCCCAGCTAATTCT and GCCTGTAATCCCAGCACCGT), No. 25 (CAGTTGTCACGGGCATCCTG and GGTCGCGGAGGGTCCTCT), No. 26 (GGCATGTTCTAGATTCACGC and TTTCCCCACCTGTTAATCA), No. 27 (GACCCTCCGCTCCAAGCTG and GATGGGCTCAAGCATGGCTG), No. 28 (GCCTGCCGAGTTCCTGCT and AGCTGCAGGTTGGgGtAATG), No. 29 (GTGGGTGGGGTAGGTG and CCTTCCTGCTTCCTTACCTT), No. 30 (AGCCAAGATCACGCCAGTA and GCAGGAGAATGGCACGAACC), No. 31 (TGTCACCAGGCGAAAC and GAATTCCCACATACACCTGC), No. 32 (CGCTGCCCACCCACTCTAGT and CTGCTTCTCCAGGCGACTGT) and No. 33 (TCCTCTGGGCT- 
CCCTACTCC and CTGGCATTACAGGCGTGAG), respectively. In these loci, No. 9, 10, 13, and 14 are disease-causing loci of $Q 0 s$ (Taniguchi et al. 1998), $J$, $Q 0 c$ and $Q 04$, respectively. Cycling conditions included an initial denaturation at $95^{\circ} \mathrm{C}$ for 9 min $30 \mathrm{~s} ; 35$ cycles at $95^{\circ} \mathrm{C}$ for $30 \mathrm{~s}, 55-65^{\circ} \mathrm{C}$ for $30 \mathrm{~s}$, and $72^{\circ} \mathrm{C}$ for $1 \mathrm{~min}$., $72^{\circ} \mathrm{C}$ for $6 \mathrm{~min}$ at the end of PCR. In some products, typing of SNP and insertion markers were executed by sequencing. The rest of the products were treated with the following restriction enzymes: AgeI (No. 25), ApaI (No. 20), ApaLI (No. 5), Asp700 (No. 21), AvaI (No. 16), BglI (No. 6), BpmI (No. 23), BsiEI (No. 14), BsrI (No. 12), EarI (No. 1), Eco0109I (No. 8), HinfI (No. 9 and No. 19), NgoIV (No. 24), NlaIII (No. 10 and No. 11), PflMI (No. 13 and No. 27), PshAI (No. 32), PvuII(No. 28), SacI (No. 7 and No. 33), Sau3A (No. 17), SphI (No. 2, No. 15 and No. 22), TaqI (No. 18), Tsp45I (No. 3). These marker loci were typed with the length of the fragments by restriction enzymes. The allele type of VNTR marker loci were decided by the length of PCR products with $2 \%$ agarose gel electrophoresis in TBE. For the typing of microsatellite marker loci, PCR products were analyzed by DNA analyzer 3100 with POP-4 capillary gel matrix (Applied Biosystems). The numbers of repeats were estimated by the length of the PCR products.

Statistical analysis Marker loci information was referred to the NCBI SNP database based on NCBI Build 34 (http://www.ncbi.nlm.nih.gov). The inferences of the frequencies of haplotypes and the individual diplotype configurations were executed by expectationmaximization (EM) algorithm (Laird 1993; Lange 2002). The tests of significances of the tables of casecontrol analysis were executed with Fisher's exact test, Pearson's $\chi^{2}$ test for test of independence, $\triangle$ TAC (Total Allele Content Difference; Collins et al. 2000), and MCMC (Markov Chain Monte Carlo; Metropolis et al. 1953; Hastings 1970; Gilks et al. 1995) methods. Because individual diplotype configurations were inferred with the EM algorithm, frequencies of haplotypes include decimal fractions. Therefore, frequencies of haplotypes were normalized to integer values for conservative direction in the tests with Fisher's exact test, $\triangle \mathrm{TAC}$, and MCMC methods. $\triangle \mathrm{TAC}$ method gave us $P$ values by approximation to $\chi_{n-1}^{2}$ distribution ( $n$ is the numbers of alleles or haplotypes). MCMC method gave us $P$ values by the sum of the probabilities of contingency tables less than given table in generating probability with the Metropolis-Hastings method. LD block was estimated by the four-gamete test (Hudson and Kaplan 1985). If the relative frequency of the fourth two-marker loci haplotype was lower than 0.05 , historical recombination was regarded not to have occurred between two-marker loci. Bayesian analysis of haplotypes for LD mapping was by BLADE (Liu et al. 2001).

\section{Results}

Identification of polymorphisms and determination of genotypes By searching for the sequence differences between samples from 94 controls and $80 A P R T$ deficient subjects, we identified 33 sequence differences within and near the $A P R T$ gene, of which 15 SNPs were already known. The locations of all such polymorphic positions, including disease-related mutations, are shown in Table 1. They were numbered from centromeric to telomeric direction, but this direction was reverse to the $A P R T$ gene because the $A P R T$ gene was coded on reverse strand. Positions No. 9, 10, 13, and 14 are those for disease-related mutations. Among the disease-related loci, No. 14 was an insertion, while all of the others, No. 9, 10, and 13 were SNPs. As described before, the mutation at No. 10 was designated $A P R T^{*} J$ that accounted for about $68 \%$ of all the disease-related mutations among Japanese (Kamatani et al. 1992). Most of the marker loci were SNPs, while 2 and 3 of them were VNTR (No. 4 and 26) and microsatellite loci (No. 29,30 , and 31), respectively.

Based on the above knowledge, we determined genotypes at 29 marker and four disease-related loci within and near the APRT gene for $80 A P R T$ patients and 94 control subjects. Table 2 shows the relative frequencies of the minor alleles for control subjects, the heterozygosities, and the relative frequencies of the genotypes at each polymorphic site (only for SNP loci). The proportions of the genotypes for each polymorphic site were in accord with the Hardy-Weinberg equilibrium in all cases (result not indicated).

Comparison of allele frequencies between APRT deficient and control subjects When the allele frequencies were compared between $A P R T$-deficient and control subjects, there were significant differences in most of the SNP markers (Table 3). $P$ values were under 0.05 for markers Nos. 1, 2, 5, 6, 7, 8, 11, 18, 19, 20, 22, 23, 24, 25, 27, 28, and 32. The minor allele frequencies were over 0.1 for markers with $P$ values under 0.05 , except for Nos. 11, 23 , and 25 . The significance of the difference as judged by the $P$ values fluctuated between markers. For markers Nos. 12, 15, and 16, significance of differences was not observed, while the minor allele frequencies of these loci were zero in the control population. Although the cases contained minor alleles for these loci, the frequencies were too low $(<0.02)$ even in the cases. This fact explains the failure of detecting significant differences between the cases and controls. For most of the loci in which relative frequencies of minor alleles were high in the cases, $P$ values tended to be low. However, $P$ value for locus No. 25 was quite low in spite of the low relative frequency of the minor allele. The evident significance is not merely a function of the location. As shown in the changes of Pearson's $\chi^{2}$ statistics (Fig. 1a) and $P$ values by MCMC (Fig. 1b) against the recombi- 
Table 1 Marker loci around and inside the APRT gene

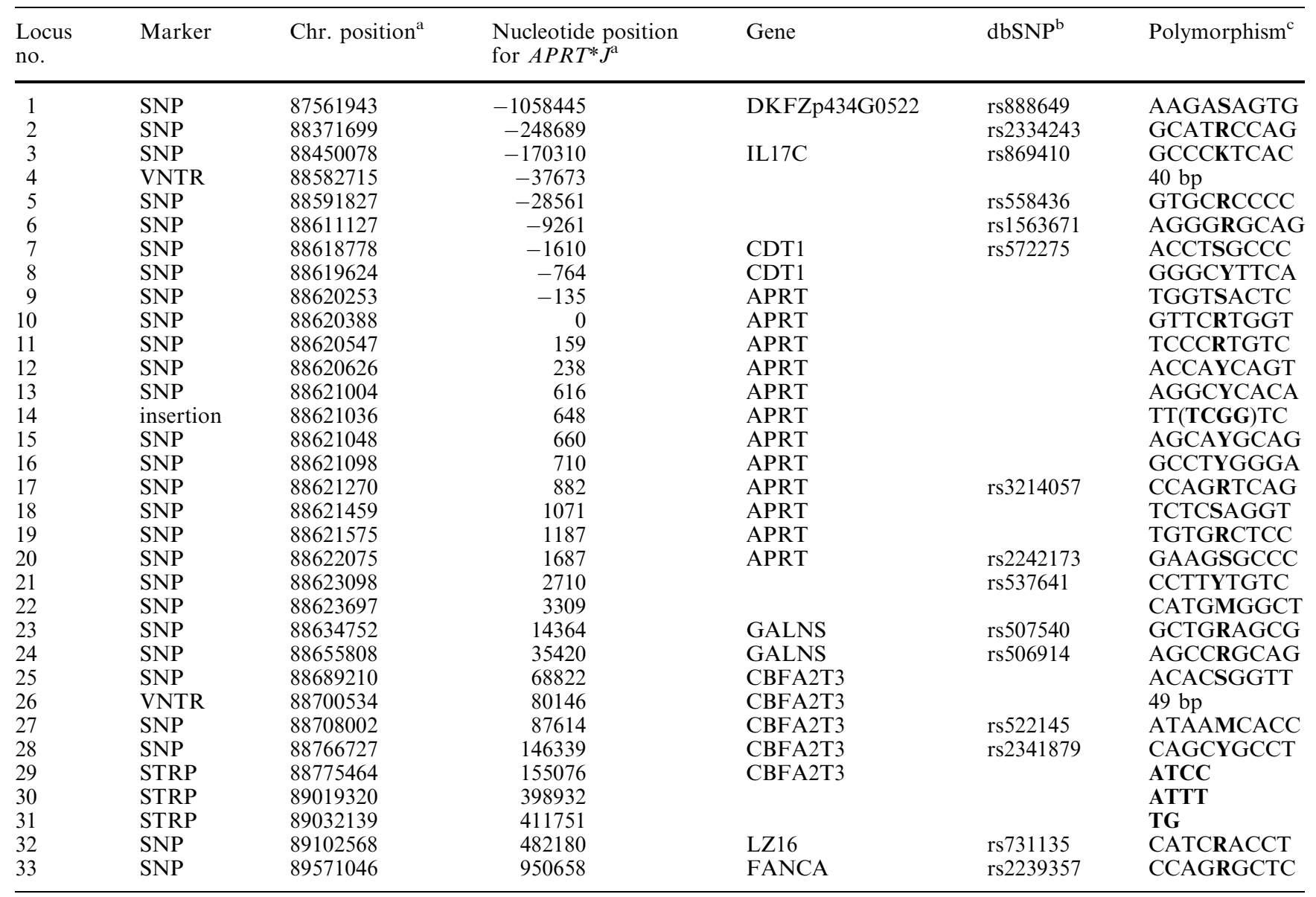

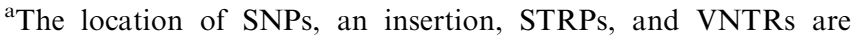
shown as chromosome position numbers based on NCBI Build 34. Locations of STRPs and VNTRs are shown as the nucleotide position at the edges of the repeats closer to $A P R T^{*} J$

nation fraction for $A P R T^{*} J$ designated locus, the relation between the significance of the difference in allele frequencies and the distance to $A P R T^{*} J$ mutation locus was not linear.

$D^{\prime}$ and $r^{2}$ between marker loci and APRT*J-causing locus in the general population LD were expected to decrease with increasing physical distance between marker loci and $A P R T^{*} J$-causing locus. We estimated $D^{\prime}$ and $r^{2}$ in the virtual population using data from control and $A P R T^{*} J$ subjects. $D^{\prime}$ and $r^{2}$ were estimated using $A P R T^{*} J$ subjects under the assumption that the population frequency of the $A P R T^{*} J$ mutation was 0.00365 (Fig. 2). The decrease of $D^{\prime}$ was not merely proportional to physical distance. In the virtual population, which consisted of $A P R T^{*} J$ and control subjects, LD between a marker locus and $A P R T^{*} J$ mutation locus was complete $\left(D^{\prime}=1\right)$ for the region between $882 \mathrm{bp}$ upstream of $A P R T^{*} J$ mutation and $1,610 \mathrm{bp}$ downstream of $A P R T^{*} J$ mutation locus, whereas $r^{2}$ was very low. This region almost accurately corresponded to the haplotype block detected as in Table 4 . The graph of $r^{2}$ was similar
${ }^{b}$ rs numbers for the SNP loci are shown as registered in dbSNP ${ }^{\mathrm{c}} \mathrm{SNPs}$ are expressed as $\mathrm{m}, \mathrm{r}, \mathrm{s}, \mathrm{y}$, and $\mathrm{k}$ for the polymorphism of AC, AG, CG, CT, and GT, respectively. Polymorphisms of STRPs and VNTRs are shown by motifs and motif length, respectively

to the graph of the $\chi^{2}$ statistic (Fig. 1a), whereas the values of $r^{2}$ were very low because of the extremely low frequency of the $A P R T^{*} J$ mutation allele.

Estimation of haplotype blocks in control subjects Haplotype block, including $A P R T^{*} J$ mutation site, was estimated by four-gamete test (Hudson and Kaplan 1985). Among marker Nos. 5, 6, 7, 8, 17, and 18 (minor allele frequency $>10 \%$ ), pairwise haplotypes and their relative frequencies were estimated by EM algorithm (Table 4). If the relative frequency of the fourth haplotype for two-marker loci was lower than 0.05 , historical recombination was regarded not to have occurred between the two-marker loci. Among marker Nos. 6, 7, 8 , and 17 , the relative frequencies of the fourth haplotypes were lower than 0.05 , whereas they were higher than 0.05 between No. 5 and others or between No. 18 and others. According to these results, it was thought that historical recombination had occurred in the sites between Nos. 5 and 6, between Nos. 17 and 18. Therefore, haplotype block, including the $A P R T^{*} J$ mutation site, occupied the region between Nos. 6 and 17 . 
Table 2 Relative frequencies of the minor alleles, heterozygosities, and relative frequencies of genotypes for SNP marker loci

\begin{tabular}{|c|c|c|c|c|c|}
\hline \multirow{2}{*}{$\begin{array}{l}\text { Locus } \\
\text { no. }\end{array}$} & \multirow{2}{*}{$\begin{array}{l}\text { Relative } \\
\text { frequency }\end{array}$} & \multirow[t]{2}{*}{ Heterozygosity } & \multicolumn{3}{|c|}{ Genotype frequency ${ }^{\mathrm{a}}$} \\
\hline & & & 11 & 12 & 22 \\
\hline 1 & 0.385 & 0.473 & 0.418 & 0.396 & 0.187 \\
\hline 2 & 0.176 & 0.289 & 0.660 & 0.330 & 0.011 \\
\hline 3 & 0.027 & 0.052 & 0.947 & 0.052 & 0.000 \\
\hline 5 & 0.457 & 0.496 & 0.340 & 0.404 & 0.255 \\
\hline 6 & 0.420 & 0.487 & 0.372 & 0.415 & 0.213 \\
\hline 7 & 0.250 & 0.375 & 0.564 & 0.372 & 0.064 \\
\hline 8 & 0.430 & 0.490 & 0.355 & 0.430 & 0.215 \\
\hline $11^{\mathrm{b}}$ & 0.000 & 0.000 & 1.000 & 0.000 & 0.000 \\
\hline $12^{\mathrm{b}}$ & 0.000 & 0.000 & 1.000 & 0.000 & 0.000 \\
\hline $15^{\mathrm{b}}$ & 0.000 & 0.000 & 1.000 & 0.000 & 0.000 \\
\hline $16^{\mathrm{b}}$ & 0.000 & 0.000 & 1.000 & 0.000 & 0.000 \\
\hline 17 & 0.186 & 0.303 & 0.681 & 0.266 & 0.053 \\
\hline 18 & 0.479 & 0.499 & 0.287 & 0.468 & 0.245 \\
\hline 19 & 0.473 & 0.499 & 0.298 & 0.457 & 0.245 \\
\hline 20 & 0.117 & 0.207 & 0.777 & 0.213 & 0.011 \\
\hline 21 & 0.160 & 0.268 & 0.745 & 0.191 & 0.064 \\
\hline 22 & 0.433 & 0.491 & 0.356 & 0.422 & 0.222 \\
\hline 23 & 0.097 & 0.175 & 0.849 & 0.108 & 0.043 \\
\hline 24 & 0.234 & 0.359 & 0.596 & 0.340 & 0.064 \\
\hline 25 & 0.011 & 0.021 & 0.978 & 0.022 & 0.000 \\
\hline 27 & 0.430 & 0.490 & 0.323 & 0.495 & 0.183 \\
\hline 28 & 0.101 & 0.182 & 0.809 & 0.181 & 0.011 \\
\hline 32 & 0.309 & 0.427 & 0.447 & 0.489 & 0.064 \\
\hline 33 & 0.074 & 0.138 & 0.851 & 0.149 & 0.000 \\
\hline
\end{tabular}

Each value is obtained from the control population

${ }^{a}$ Alleles 1 and 2 denote the major allele and minor alleles, respectively, at each locus

${ }^{\mathrm{b}}$ Polymorphisms were not observed in the control subjects at Nos. $11,12,15$ and 16 loci

Haplotype inference We then inferred haplotypes for the region extending from Nos. 6-17 that was defined as haplotype block for the control subjects using common SNPs (minor allele frequency $>10 \%$ ). Table 5 shows that the majority $(95 \%)$ of haplotypes were accounted for by five haplotypes in the control chromosomes. Haplotype heterozygosity was 0.562 . Among the chromosomes from APRT-deficient subjects, the majority of haplotypes were accounted for by only four different haplotypes, and the haplotype heterozygosity was 0.555 . When the frequency of each haplotype was compared between $A P R T$-deficient and control subjects, the frequency of haplotype No. 2 was much higher among the $A P R T$-deficient subjects than the control subjects. When the significance of the difference in haplotype frequencies between $A P R T$-deficient and control subjects was compared, $P$ values by Fisher's exact test, $\triangle$ TAC, and MCMC methods were $9.74 \times 10^{-23}, 1.29 \times 10^{-9}$ $(\triangle \mathrm{TAC}=47.34)$, and $<10^{-6}$, respectively. When the haplotype inference was performed using only the $A P R T^{*} J$ chromosomes, the number of major haplotypes was two, while the haplotype heterozygosity was 0.133 . In fact, the haplotype No. 2 that was present at a much higher frequency among the $A P R T$-deficient chromosomes than the control chromosomes was the haplotype to which almost all the chromosomes containing $A P R T^{*} J$ belonged. When the significance of the
Table 3 Significance of the difference in allele frequencies between $A P R T$-deficient and control subjects at SNP marker loci

\begin{tabular}{|c|c|c|}
\hline Locus no. & $\begin{array}{l}\text { Pearson's } \chi^{2} \text { test }^{\mathrm{a}} \\
P \text { value }\end{array}$ & $\begin{array}{l}\mathrm{MCMC}^{\mathrm{a}} \\
P \text { value }\end{array}$ \\
\hline 1 & $2.46 \times 10^{-5}$ & $5.60 \times 10^{-5}$ \\
\hline 2 & $1.70 \times 10^{-5}$ & $1.00 \times 10^{-5}$ \\
\hline 3 & $5.62 \times 10^{-1}$ & $7.60 \times 10^{-1}$ \\
\hline 5 & $2.30 \times 10^{-2}$ & $2.85 \times 10^{-2}$ \\
\hline 6 & $0.00^{\mathrm{b}}$ & $<10^{-6}$ \\
\hline 7 & $7.77 \times 10^{-16}$ & $<10^{-6}$ \\
\hline 8 & $0.00^{\mathrm{b}}$ & $<10^{-6}$ \\
\hline 11 & $7.40 \times 10^{-3}$ & $9.32 \times 10^{-3}$ \\
\hline 12 & $1.24 \times 10^{-1}$ & $2.12 \times 10^{-1}$ \\
\hline 15 & $2.78 \times 10^{-1}$ & $4.60 \times 10^{-1}$ \\
\hline 16 & $1.24 \times 10^{-1}$ & $2.12 \times 10^{-1}$ \\
\hline 17 & $1.15 \times 10^{-1}$ & $1.22 \times 10^{-1}$ \\
\hline 18 & $2.45 \times 10^{-3}$ & $2.87 \times 10^{-3}$ \\
\hline 19 & $2.08 \times 10^{-11}$ & $<10^{-6}$ \\
\hline 20 & $7.80 \times 10^{-6}$ & $2.00 \times 10^{-6}$ \\
\hline 21 & $9.10 \times 10^{-2}$ & $1.03 \times 10^{-1}$ \\
\hline 22 & $1.29 \times 10^{-14}$ & $<10^{-6}$ \\
\hline 23 & $1.91 \times 10^{-4}$ & $2.29 \times 10^{-4}$ \\
\hline 24 & $1.51 \times 10^{-13}$ & $<10^{-6}$ \\
\hline 25 & $0.00^{\mathrm{b}}$ & $<10^{-6}$ \\
\hline 27 & $2.11 \times 10^{-15}$ & $<10^{-6}$ \\
\hline 28 & $3.54 \times 10^{-5}$ & $2.00 \times 10^{-6}$ \\
\hline 32 & $3.17 \times 10^{-3}$ & $4.19 \times 10^{-3}$ \\
\hline 33 & $8.20 \times 10^{-2}$ & $9.95 \times 10^{-2}$ \\
\hline
\end{tabular}

difference in haplotype frequencies between $A P R T^{*} J$ and control subjects was compared, $P$ values by Fisher's exact test, $\triangle \mathrm{TAC}$, and MCMC methods were $1.92 \times 10^{-34}, 3.55 \times 10^{-15}(\Delta \mathrm{TAC}=73.83)$, and $<10^{-6}$, respectively.

When the number of haplotypes was estimated using disease chromosomes, it was fewer for disease chromosomes than control chromosomes. The haplotype heterozygosity was lower for disease chromosomes than for control chromosomes. Although the above characteristics are the most prominent, when all the disease chromosomes were of a single origin, they were still detectable even when the disease chromosomes were derived from a few origins. The test of the difference in frequency distributions of haplotypes between case and control chromosomes may be possible by the Fisher's exact test, $\triangle \mathrm{TAC}$, and MCMC methods.

Comparison of allele frequencies for STRP and VNTR markers The comparison of allele frequencies at multiallelic loci is generally more complicated than SNP loci. We tested the significances for independence of allele distributions between $A P R T$-deficient subjects and control subjects with Fisher's exact test, $\triangle \mathrm{TAC}$ values method, and MCMC method testing for the independence of $2 \times m$ contingency tables. We found three STRP and two VNTR marker loci, one locating downstream of $A P R T$ gene (No. 4) and the other four upstream of the 

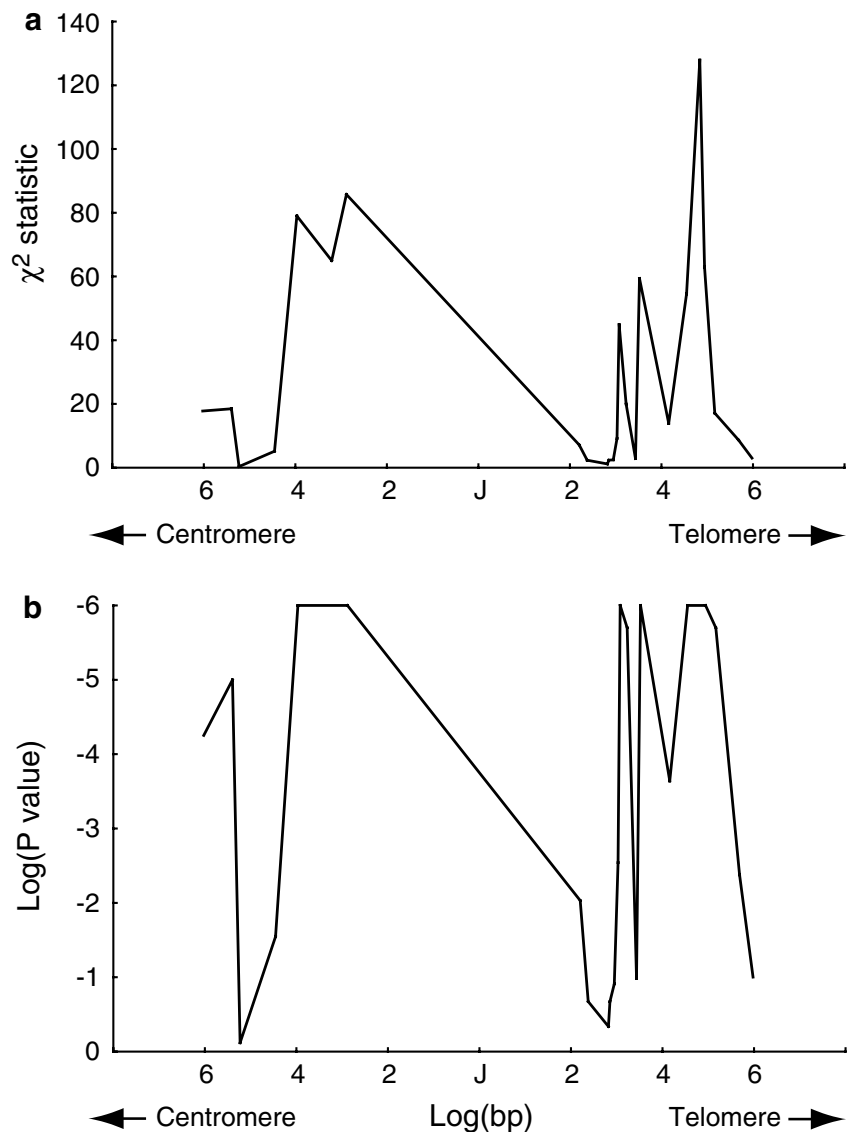

Fig. 1a,b Pearson's $\chi^{2}$ statistics and logarithm of $P$ values from MCMC to test differences in allele frequencies between cases and controls. a Pearson's $\chi^{2}$ statistics. b Logarithm of $P$ values by MCMC method. Horizontal axis shows the locations of the marker loci as shown by the logarithm of physical distances (bp) from the $A P R T^{*} J$ mutation locus $(\mathrm{J})$

gene (Nos. 26, 29, 30, and 31). At No. 4 VNTR locus, 97.4\% (152/156) of all APRT-deficient chromosomes had the 11 repeat alleles, while the 11 repeat alleles were observed in only $69.4 \%(129 / 186)$ of control chromosomes (Table 6 ). $P$ values by three tests indicated that 11 repeat alleles at No. 4 locus were significantly linked to $A P R T$-deficient mutations. As will be stated later in this manuscript, all chromosomes containing the $A P R T^{*} J$ mutation had the same 11 repeat polymorphism at the No. 4 locus (Table 8). This result indicated that the $A P R T^{*} J$ mutation was completely linked to a single 11repeat polymorphism at the No. 4 VNTR locus locating downstream of the APRT gene.

For the upstream STRP and VNTR loci, $P$ values for the differences in allele distributions between $A P R T$ deficient (case) and control subjects were less than the general significance level (0.05), except for the No. 26 marker locus (Table 7). $P$ values for the difference between $A P R T^{*} J$ and control subjects were lower than between case and control subjects for these marker loci. Here, APRT-deficient subjects include those with $A P R T^{*} J$ and other mutant alleles. Therefore, the tests for the subjects with multiple mutant alleles resulted in
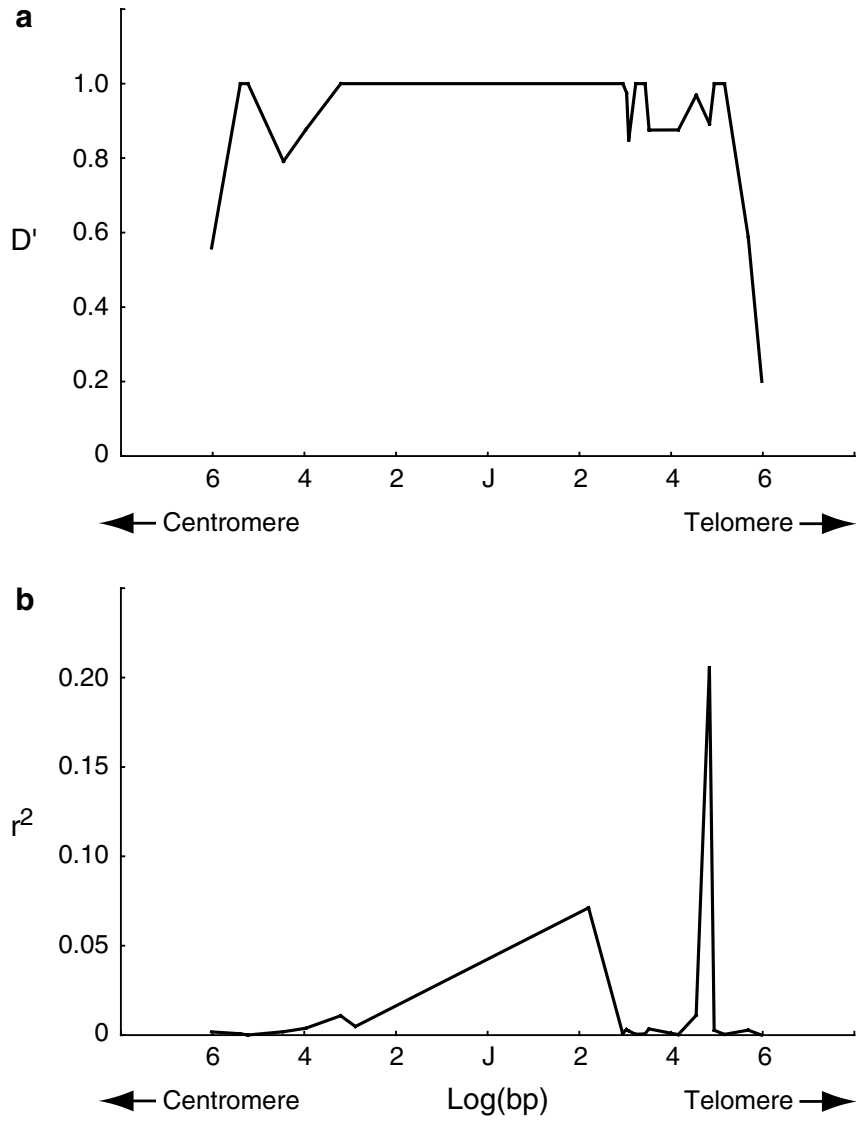

Fig. 2a,b $D^{\prime}$ and $r^{2}$ between the $A P R T^{*} J$ mutation locus and a marker locus. $A P R T^{*} J$ allele frequency was assumed to be 0.00365 . a $D^{\prime}$. b $r^{2}$. Horizontal axis shows the locations of the loci as shown by the logarithm of physical distances (bp) from the $A P R T^{*} J$ mutation locus $(\mathrm{J})$

higher $P$ values than the test for the subjects with a single mutation. For upstream STRP and VNTR loci, $P$ values were higher for loci (Nos. 26 and 29) closer to the $A P R T$ gene than father loci (Nos. 30 and 31). However, the higher $P$ values in closer loci do not indicate a weaker LD but seem to be caused by low heterozygosities at those loci in control subjects. Those results indicate that the analysis of STRP or VNTR loci with high heterozygosity in control subjects is necessary to detect disease-related loci efficiently.

Linkage between APRT deficiency mutations and repeat markers Haplotypes were inferred using one of the APRT mutation loci and a repeat marker locus. The results are shown in Table 8. The downstream VNTR locus No. 4 was in complete LD with the $A P R T^{*} J$ mutation (including $J$ homo and $J / Q O$ hetero), and a single 11 repeat polymorphism was completely linked to $A P R T^{*} J$ mutation. The $Q 0$ mutation was nearly completely linked to the same 11 repeat. For the upstream marker locus No. 30, the most frequent allele linked to $A P R T^{*} Q 0$ mutation was different from the most frequent allele linked to nonmutation. On the other hand, the most frequent allele linked to $A P R T^{*} J$ mutation was 
Table 4 Haplotype blocks inferred by four-gamete test. Pairwise haplotypes were constructed by two-marker loci between Nos. 5, 6, 7, 8, 17 and 18 in control subjects. For all pair of loci, possible four haplotypes and their relative frequencies were estimated by EM algorithm. Each cell contains four haplotypes and their relative frequencies

\begin{tabular}{|c|c|c|c|c|c|c|c|c|c|c|}
\hline $\begin{array}{l}\text { Locus } \\
\text { no. }\end{array}$ & 5 & & 6 & & 7 & & 8 & & 17 & \\
\hline \multirow[t]{4}{*}{6} & GG & 0.391 & & & & & & & & \\
\hline & AA & 0.269 & & & & & & & & \\
\hline & $\mathrm{AG}$ & 0.189 & & & & & & & & \\
\hline & GA & 0.152 & & & & & & & & \\
\hline \multirow[t]{4}{*}{7} & GC & 0.453 & GC & 0.554 & & & & & & \\
\hline & $\mathrm{AC}$ & 0.297 & $\mathrm{AG}$ & 0.224 & & & & & & \\
\hline & $\mathrm{AG}$ & 0.161 & $\mathrm{AC}$ & 0.196 & & & & & & \\
\hline & GG & 0.089 & GG & $0.026^{\mathrm{a}}$ & & & & & & \\
\hline \multirow[t]{4}{*}{8} & GC & 0.387 & GC & 0.547 & $\mathrm{CC}$ & 0.561 & & & & \\
\hline & AT & 0.273 & AT & 0.398 & GT & 0.243 & & & & \\
\hline & $\mathrm{AC}$ & 0.185 & GT & 0.033 & $\mathrm{CT}$ & 0.189 & & & & \\
\hline & GT & 0.155 & $\mathrm{AC}$ & $0.022^{\mathrm{a}}$ & GC & $0.007^{\mathrm{a}}$ & & & & \\
\hline \multirow[t]{4}{*}{17} & GA & 0.472 & GA & 0.567 & $\mathrm{CA}$ & 0.564 & CA & 0.561 & & \\
\hline & AA & 0.342 & AA & 0.247 & GA & 0.250 & TA & 0.253 & & \\
\hline & $\mathrm{AG}$ & 0.115 & $\mathrm{AG}$ & 0.173 & $\mathrm{CG}$ & 0.186 & TG & 0.180 & & \\
\hline & GG & 0.071 & GG & $0.013^{\mathrm{a}}$ & GG & $0.000^{\mathrm{a}}$ & CG & $0.007^{\mathrm{a}}$ & & \\
\hline \multirow[t]{4}{*}{18} & GG & 0.316 & GG & 0.344 & $\mathrm{CC}$ & 0.411 & $\mathrm{CG}$ & 0.348 & $\mathrm{AG}$ & 0.514 \\
\hline & $\mathrm{AC}$ & 0.252 & $\mathrm{AC}$ & 0.243 & $\mathrm{CG}$ & 0.339 & $\mathrm{TC}$ & 0.257 & $\mathrm{AC}$ & 0.300 \\
\hline & GC & 0.227 & GC & 0.236 & GG & 0.183 & $\mathrm{CC}$ & 0.222 & GC & 0.179 \\
\hline & $\mathrm{AG}$ & 0.206 & $\mathrm{AG}$ & 0.177 & GC & 0.067 & TG & 0.173 & GG & $0.007^{\mathrm{a}}$ \\
\hline
\end{tabular}

${ }^{a}$ If the relative frequency of the fourth haplotype was less than 0.05 , it was regarded that historical recombination had not occurred between the pair of the marker loci. By this test, the region between Nos. 6 and 17 was recognized as a haplotype block

Table 5 Frequencies of haplotypes within the haplotype block in the $A P R T$ gene and haplotype heterozygosities in $A P R T^{*} J, A P R T$ deficient, and control subjects. Haplotype block (Nos. 6, 7, 8 and 17) was constructed using the data from control subjects in Table 4 ,
Haplotypes with higher frequencies were collected until the accumulated frequencies became over $95 \%$, and only such common haplotypes were shown

\begin{tabular}{|c|c|c|c|c|c|c|}
\hline & \multirow[t]{2}{*}{ Haplotype no. } & \multirow[t]{2}{*}{ Haplotype } & \multicolumn{3}{|c|}{ Frequency $^{\mathrm{a}}$} & \multirow[t]{2}{*}{ Heterozygosity } \\
\hline & & & Inferred & Relative & Normalized $^{\mathrm{b}}$ & \\
\hline $\mathrm{APRT}^{*} \mathrm{~J}$ & 4 & ggta & 6.00 & 0.071 & 6 & 0.133 \\
\hline \multirow{2}{*}{ Case } & 2 & agta & 99.59 & 0.622 & 99 & \multirow{2}{*}{0.555} \\
\hline & 3 & actg & 36.00 & 0.225 & 36 & \\
\hline \multirow[t]{5}{*}{ Control } & 1 & gcca & 100.62 & 0.535 & 100 & \multirow[t]{5}{*}{0.562} \\
\hline & 2 & agta & 39.95 & 0.213 & 40 & \\
\hline & 3 & actg & 31.14 & 0.166 & 32 & \\
\hline & 4 & ggta & 5.26 & 0.028 & 6 & \\
\hline & 5 & acca & 3.07 & 0.016 & 3 & \\
\hline
\end{tabular}

${ }^{\mathrm{a}}$ Frequencies were inferred by EM algorithm

${ }^{\mathrm{b}}$ Normalized frequencies indicate the integers normalized from inferred values

different from the most frequent allele linked to nonmutation at No. 31. If a mutation causative of disease had occurred on the chromosome with low frequency marker allele, "the low frequency allele" linked to the mutation has spread on the case population. The marker loci Nos. 30 and 31 should be examples. These results indicate that chromosomes containing a common disease-causing mutation tend to have a common polymorphism at a nearby locus.

Bayesian analysis of haplotypes for linkage disequilibrium mapping Since the APRT-deficient chromosomes have multiple ancestral haplotypes, it is of interest to analyze the data using BLADE (Liu et al. 2001), a recently released software for the Bayesian analysis of haplotypes for LD mapping. Figure 3 indicates the results obtained when the data for both $A P R T$-deficient and control chromosomes were applied to the software. For this study, all data for the disease-related loci were removed. When the data for the control chromosomes were applied to the software, the posterior probabilities of the mutation clusters to which each current haplotype belongs tended to be dispersed (Fig. 3d). In contrast, when BLADE was applied with $A P R T$-deficient chromosomes, $A P R T^{*} J$ and $A P R T^{*} Q O c$ chromosomes were separated from the other mutation chromosomes if the number of clusters was up to four (Fig. 3a,b). $A P R T^{*} Q 04$ chromosomes were separated from 'null' when the number of clusters became five (Fig. 3c). However, $A P R T^{*} J$ chromosomes were not concentrated in a single cluster. This may reflect the fact that the $A P R T^{*} J$ mutation is older than $A P R T^{*} Q O$ mutations, and $A P R T^{*} J$ chromosomes were judged to have two 
Table 6 Allele number of repeat marker loci and heterozygosity in $A P R T^{*} J, A P R T$-deficient and control subjects

\begin{tabular}{|c|c|c|c|c|c|c|c|c|}
\hline \multirow[t]{2}{*}{ Locus no. } & \multicolumn{7}{|c|}{ Contingency table ${ }^{\mathrm{a}}$} & \multirow[t]{2}{*}{ Heterozygosity } \\
\hline & Repeat & 11 & 13 & 8 & 6 & 7 & 10 & \\
\hline \multirow[t]{4}{*}{4} & $A P R T^{*} J$ & 82 & 0 & 0 & 0 & 0 & 0 & 0.000 \\
\hline & Case & 152 & 2 & 0 & 2 & 0 & 0 & 0.050 \\
\hline & Control & 129 & 27 & 19 & 9 & 1 & 1 & 0.485 \\
\hline & Repeat & 3 & 2 & 4 & & & & \\
\hline \multirow[t]{4}{*}{26} & $A P R T^{*} J$ & 79 & 5 & 0 & & & & 0.113 \\
\hline & Case & 153 & 7 & 0 & & & & 0.084 \\
\hline & Control & 168 & 18 & 2 & & & & 0.192 \\
\hline & Repeat & 12 & 11 & 13 & 10 & 14 & & \\
\hline \multirow[t]{4}{*}{29} & $A P R T^{*} J$ & 80 & 4 & 0 & 0 & 0 & & 0.091 \\
\hline & Case & 147 & 10 & 3 & 0 & 0 & & 0.152 \\
\hline & Control & 153 & 19 & 7 & 7 & 2 & & 0.325 \\
\hline & Repeat & 14 & 13 & 12 & 9 & 15 & 11 & \\
\hline \multirow[t]{4}{*}{30} & $A P R T^{*} J$ & 57 & 6 & 8 & 12 & 1 & 0 & 0.504 \\
\hline & Case & 70 & 51 & 17 & 16 & 2 & 4 & 0.685 \\
\hline & Control & 69 & 49 & 49 & 12 & 6 & 1 & 0.718 \\
\hline & Repeat & 17 & 18 & 20 & 21 & 16 & 19 & \\
\hline \multirow[t]{3}{*}{31} & $A P R T^{*} J$ & 20 & 52 & 9 & 1 & 0 & 0 & 0.526 \\
\hline & Case & 75 & 63 & 17 & 1 & 0 & 2 & 0.604 \\
\hline & Control & 110 & 37 & 28 & 8 & 2 & 1 & 0.586 \\
\hline
\end{tabular}

${ }^{\mathrm{a}}$ The differences in allele frequencies between symptomatic ( $A P R T^{*} J$ or $A P R T$-deficient) and control subjects were tested
Table 7 Significance of difference in allele frequencies between $A P R T^{*} J$ or $A P R T$-deficient and control subjects

\begin{tabular}{|c|c|c|c|c|}
\hline Locus no. & & $\begin{array}{l}\text { Fisher's exact } \\
\text { test }^{\mathrm{a}}\end{array}$ & $\Delta \mathrm{TAC}^{\mathrm{a}}$ & $\mathrm{MCMC}^{\mathrm{a}}$ \\
\hline \multirow[t]{2}{*}{4} & $A P R T^{*} J$ & $2.01 \times 10^{-8}$ & $1.10 \times 10^{-5}$ & $<10^{-6}$ \\
\hline & Case & $5.59 \times 10^{-12}$ & $3.50 \times 10^{-5}$ & $<10^{-6}$ \\
\hline \multirow[t]{2}{*}{26} & $A P R T^{*} J$ & $4.70 \times 10^{-1}$ & $9.60 \times 10^{-2}$ & $3.69 \times 10^{-1}$ \\
\hline & Case & $5.58 \times 10^{-2}$ & $4.37 \times 10^{-2}$ & $4.46 \times 10^{-2}$ \\
\hline \multirow[t]{2}{*}{29} & $A P R T^{*} J$ & $2.91 \times 10^{-2}$ & $7.77 \times 10^{-3}$ & $2.74 \times 10^{-2}$ \\
\hline & Case & $1.46 \times 10^{-2}$ & $3.29 \times 10^{-2}$ & $1.40 \times 10^{-2}$ \\
\hline \multirow[t]{2}{*}{30} & $A P R T^{*} J$ & $1.87 \times 10^{-7}$ & $2.87 \times 10^{-7}$ & $<10^{-6}$ \\
\hline & Case & $1.80 \times 10^{-3}$ & $3.35 \times 10^{-3}$ & $1.56 \times 10^{-3}$ \\
\hline \multirow[t]{2}{*}{31} & $A P R T^{*} J$ & $2.57 \times 10^{-10}$ & $2.90 \times 10^{-8}$ & $<10^{-6}$ \\
\hline & Case & $1.95 \times 10^{-4}$ & $9.19 \times 10^{-4}$ & $2.20 \times 10^{-4}$ \\
\hline
\end{tabular}

${ }^{\mathrm{a}}$ The differences in allele frequencies between symptomatic ( $A P R T^{*} J$ or $A P R T$-deficient) and control subjects were tested by Fisher's exact test, $\triangle \mathrm{TAC}$, and MCMC methods origins. On the other hand, $A P R T^{*} Q O c$ and $Q 04$ chromosomes were concentrated in single clusters. Therefore, BLADE software successfully clustered the $A P R T^{*} Q O c$ and $Q 04$ chromosomes according to the disease-related mutations without the direct data of the disease-related mutations, whereas $A P R T^{*} J$ chromosomes were not successfully clustered. When the maximum likelihood of the data was calculated assuming varying numbers of clusters (excluding the null cluster), the curves showing the relation between the number of clusters and the maximum likelihood were quite different for $A P R T$ deficient and control chromosomes. Thus, when data from the APRT-deficient chromosomes were used, the maximum likelihood increased, with increasing assumed number of clusters as long as the number of clusters was not more than three, and it stayed at the same level at assumed cluster numbers over three (Fig. 4). In contrast,

Table 8 Linkage between $A P R T$ deficiency mutation and repeat markers. Each cell contains the motif repeats in alleles and their frequencies. Frequencies were inferred by EM algorithm

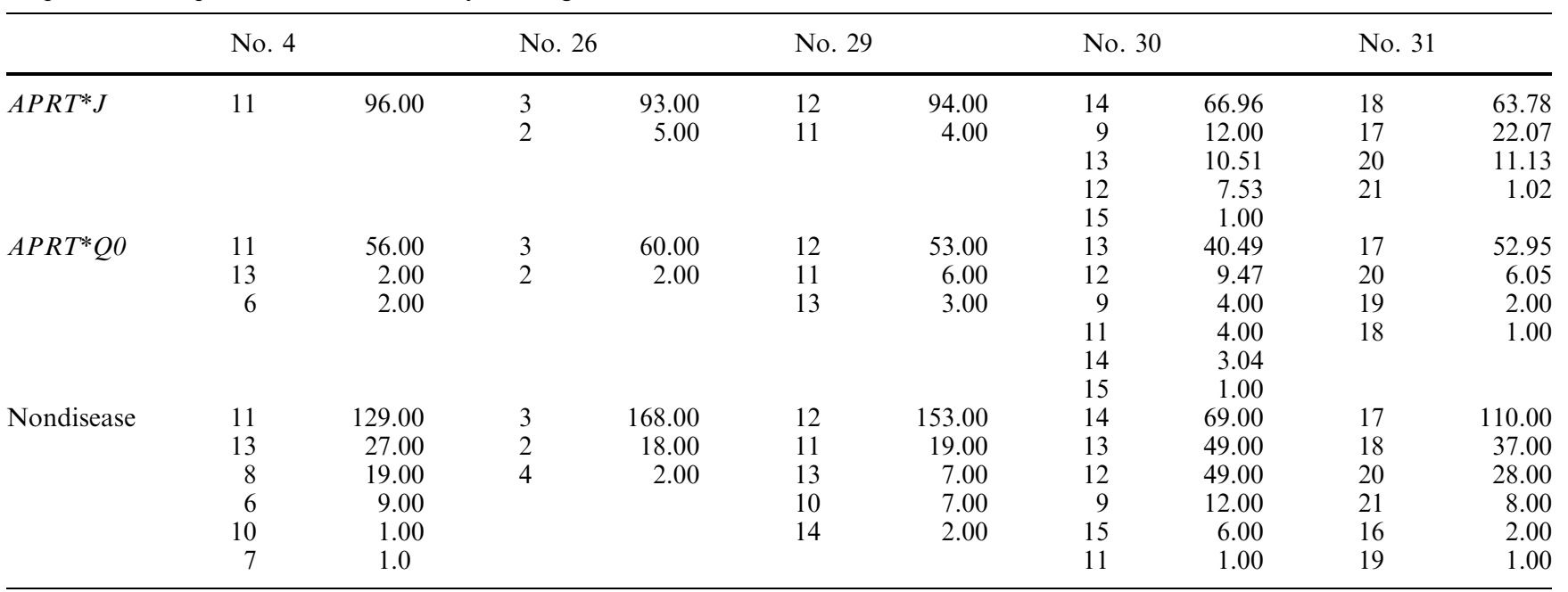


Fig. 3a-d Affiliation of APRTdeficient and control chromosomes derived from the analysis with BLADE.

Haplotypes of chromosomes were constructed with 16 SNP marker loci with minor allele frequencies over 0.1 (Nos. 1, 2 , $5,6,7,8,17,18,19,20,21,22$, 24, 27, 28, and 32). APRTdeficient chromosomes were clustered to a three, b four, and c five clusters, while control chromosomes were clustered to d seven clusters. Each vertical bar indicates a chromosome. $J$, $c, s, 4$ and $u$ in horizontal axis denote $A P R T^{*} J, A P R T^{*} Q 0 c$, $A P R T^{*} Q 0 s, A P R T^{*} Q 04$, and $A P R T^{*} Q O u$, respectively. Some chromosomes were clustered to the "null" cluster a

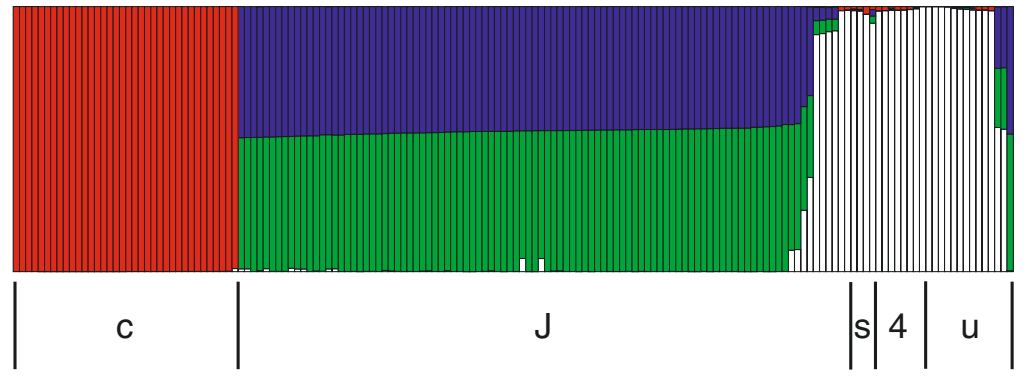

b

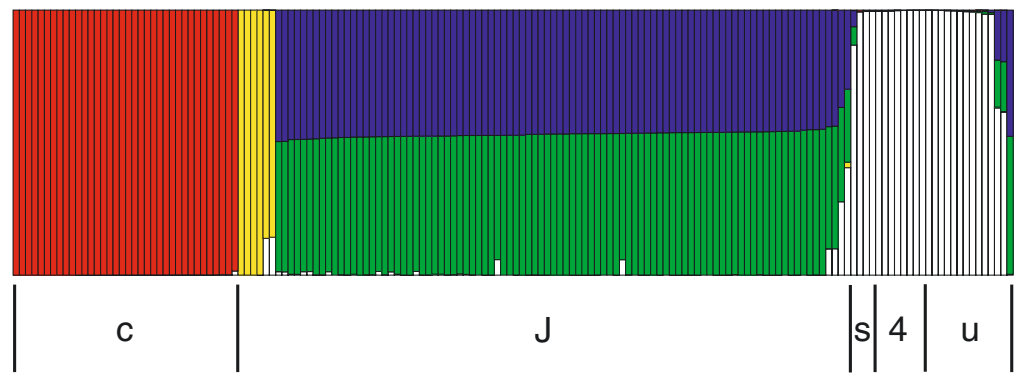

c

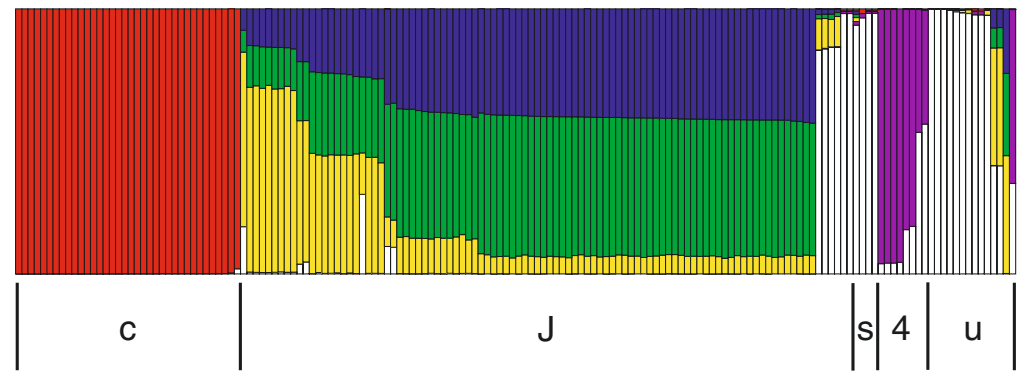

d

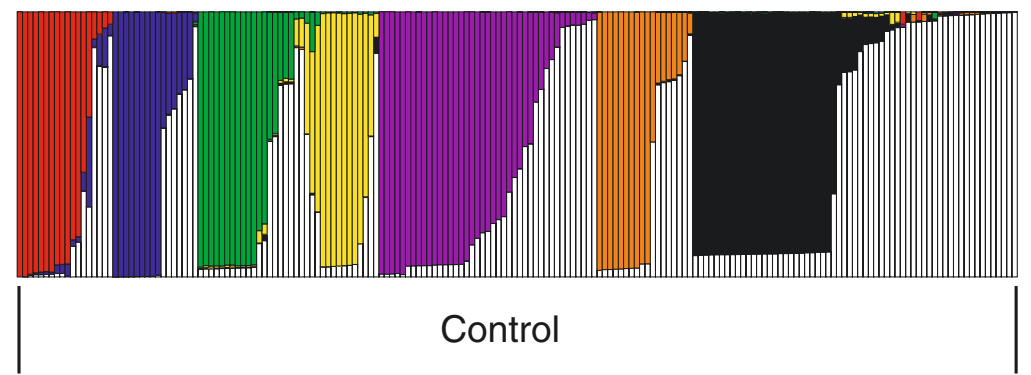

cluster1

cluster2

cluster3

$\square$ cluster4

cluster5

cluster6

cluster7

$\square$ null the maximum likelihood for the data from control chromosomes increased with the increasing number of clusters of over three. These results indicate that the suitable cluster number in $A P R T$-deficient chromosomes was three, whereas the suitable cluster number in control chromosomes was not decided.

\section{Discussion}

Common disease-common variant hypothesis assumes that the majority of the disease-related mutations for a common disease are accounted for by a limited number of the common variants derived from common origins. If this hypothesis is right, $\mathrm{LD}$ is expected to be present between the disease-related loci and flanking marker loci. The disease chromosomes are expected to be derived from a limited number of ancestral haplotypes concerning the disease-related region. Based on this hypothesis, genome-wide studies searching for diseaserelated genes for common diseases are now under way. Such studies are not intended to identify disease-related mutations directly but to encounter some marker loci that are in LD with the disease-related loci.

However, it is not clear whether this approach can successfully identify disease-related genes for common diseases. Even if it can, the optimal conditions of such investigations remain to be clarified. The questions include what types of markers are to be used, how large the distance between markers should be, and whether 


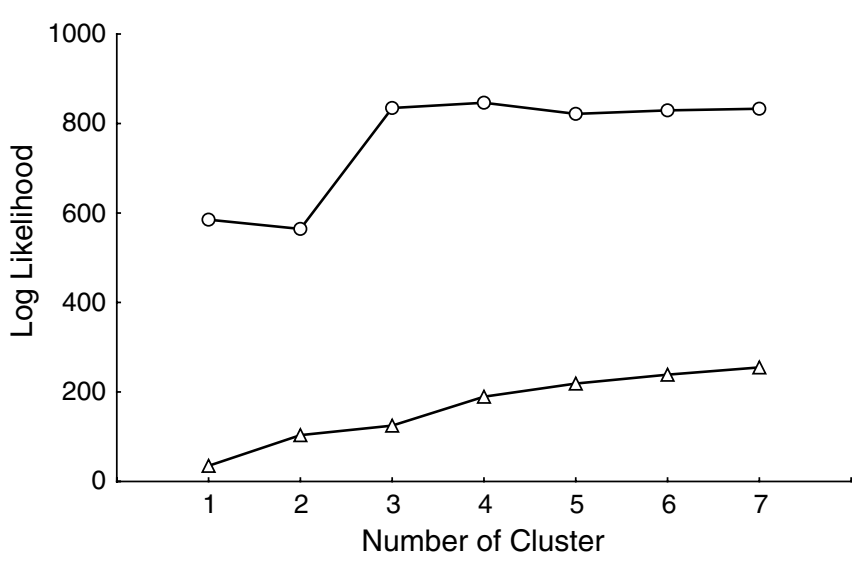

Fig. 4 Maximum log likelihood for the numbers of clusters. Circle and triangle indicate $A P R T$-deficient and control chromosomes, respectively. The log likelihoods are output results from BLADE

the marker loci should be concentrated in the genes. We could use SNPs, STRPs, and VNTR markers, each of which has different characteristics. SNPs are present at the highest frequency, while a single STRP locus has multiple alleles. The mutation rates for SNPs are likely to be very low, while those for STRPs may be rather high.

Our results showed that the comparison of the allele frequencies at marker loci between $A P R T$-deficient and control subjects was an effective method to identify loci that are in LD with the true disease-related loci. Although SNPs were present at the highest frequency, both STRP and VNTR may serve as markers by which the true disease-related loci can be identified, because the frequency distributions of the alleles at both STRP and VNTR loci were significantly different between $A P R T$ deficient and control subjects when the statistic analysis was performed by the Fisher's exact test, $\triangle \mathrm{TAC}$, and MCMC method.

In the analyses with STRP and VNTR loci, $P$ values for the tests of the differences in the frequencies at single loci were not sufficiently low when the heterozygosities were low. However, the marker loci with high heterozygosities generally gave significant difference. Especially at No. 4 VNTR locus that is $37.7 \mathrm{kbp}$ apart from the $A P R T^{*} J$ mutation locus, 11 repeat polymorphism was completely linked to $A P R T^{*} J$ mutation (Table 8). At Nos. 30 and 31 loci, very low $P$ values were also obtained. The most frequent alleles were different at Nos. 30 and 31 loci between $A P R T^{*} Q 0$ and $J$ mutation chromosomes. The above evidence suggests that LD analysis to search for disease-causing loci is feasible in the population of common origin.

Comparison of frequencies of marker alleles of SNPs between affected and control subjects are also likely to be a sensitive method to identify candidate markers associated with the disease-related loci. $P$ values tended to be low for the markers with high heterozygosities (Tables 2,3). Even if the marker loci are close to the disease-related loci, the evident significance was not shown when the frequencies of the minor alleles were low (Fig. 1, Table 3). Even though some marker loci were in the complete $\operatorname{LD}\left(D^{\prime}=1\right)$ with the disease loci, significant $P$ values were not observed for those loci (Figs. 1, 2a). However, loci with low heterozygosities (No. 25, for example) occasionally gave significant $P$ values. These results suggest that $P$ values in singlemarker loci do not always reflect $D^{\prime}$ between diseaserelating and marker loci. On the other hand, the $r^{2}$ values were extremely low as though the values denied the LD. Both $D^{\prime}$ and $r^{2}$ are insufficient as measure of LD when the haplotype frequencies are in extreme imbalance.

When we performed a haplotype analysis using SNP, haplotypes had to be constructed within haplotype blocks for the inferred haplotypes to be reliable. A haplotype block with a size of $10.1 \mathrm{kbp}$ was observed using the data from the control chromosomes by fourgamete test. This haplotype block did not cover the entire APRT gene. When the haplotypes were inferred by the EM algorithm-based method, the frequencies of haplotype as well as the most frequent haplotypes were different between the APRT-deficient and control chromosomes (Table 5). Therefore, comparison of frequencies of haplotypes between case and control chromosomes is likely to serve as an effective method to identify disease-related loci.

Recently-released BLADE algorithm for the Bayesian analysis of haplotypes for LD mapping is of interest because of its flexibility. The analysis of our data using the algorithm indicated that it may serve as a powerful method to identify disease-related chromosomes from marker genotypes. Thus, when the data from the $A P R T$ deficient chromosomes were applied, $A P R T^{*} Q 0 c$ and Q04 chromosomes were concentrated in single clusters, whereas $A P R T^{*} J$ chromosomes were not concentrated in a single cluster (Fig. 3). This split of $A P R T^{*} J$ chromosomes' affiliation may reflect the fact that $A P R T^{*} J$ mutation is older than $A P R T^{*} Q O$ mutations and original single haplotype may have been split into two by recombination in $A P R T^{*} J$ chromosomes. Therefore, $A P R T^{*} J$ chromosomes had been concentrated in a single cluster when the data from a more narrow region (Nos. 2-32) were applied to BLADE (data not shown). When BLADE software is applied, data from suitablelength regions should be used. If suitable data are used, this algorithm could successfully estimate disease-related chromosomes without the data of disease-related loci as shown in $A P R T^{*} Q 0 c$ and $Q 04$.

Although APRT deficiency is not a common disease, the combined frequency of the disease-related mutations is among the highest of all the genetic diseases in the Japanese. Data obtained from the analysis of LD and haplotypes related to this genetic deficiency may lead to a better understanding of the mode of LD and haplotypes and provide valuable information about the relationship between disease-related mutations and LD in common diseases in general. Our results suggest that the comparison of marker allele frequencies between case 
and control chromosomes is a sensitive method to list up candidates while the analysis of haplotypes using EMbased haplotype inference and the Bayesian method may serve as tests to examine whether the candidate regions are real disease-related loci.

Acknowledgements This study was supported by a grant from Research for the Future Program from the Japan Society for the Promotion of Science (JSPS).

\section{References}

Angius A, Petretto E, Maestrale GB, Forabosco P, Casu G, Piras D, Fanciulli M, Falchi M, Melis PM, Palermo M, Pirastu M (2002) A new essential hypertension susceptibility locus on chromosome 2 p24-2p25, detected by genomewide seach. Am J Hum Genet 71:893-905

Cartier P, Hamet M, Hamburger J (1974) Une nouvelle maladie metabolique: le deficit en adenine phosphoribosyltransferase avec lithiase de 2,8-dihydroxyadenine. CR Seances Acad Sci 279:883-886

Collins HE, Li H, Inda SE, Anderson J, Laiho K, Tuomilehto J, Seldin MF (2000) A simple and accurate method for determination of microsatellite total allele content differences between DNA pools. Hum Genet 106:218-226

Debray H, Cartier P, Temstet A, Cendron J (1976) Child's urinary lithiasis revealing a complete deficit in adenine phosphoribosyl transferase. Pediatr Res 10:762-766

Fratini A, Simmers RN, Callen DF, Hyland VJ, Tischfield JA, Stambrook PJ, Sutherland GR (1986) A new location for the human adenine phosphoribosyltransferase gene (APRT) distal to the haptoglobin (HP) and fra(16)(q23) (FRA16D) loci. Cytogenet Cell Genet 43:10-13

Gilks WR, Richardson S, Spiegelhalter DJ (1995) Markov Chain Monte Carlo in practice. Chapman \& Hall, London

Hastings WK (1970) Monte Carlo sampling methods using Markov chains and their applications. Biometrika 57:97-109

Henderson JF, Kelley WN, Rosenbloom FM, Seegmiller JE (1969) Inheritance of purine phosphoribosyltransferases in man. Am J Hum Genet 21:61-70

Hidaka Y, Tarle SA, Fujimori S, Kamatani N, Kelley WN, Palella TD (1988) Human adenine phosphoribosyltransferase deficiency: demonstration of a single mutant allele common to the Japanese. J Clin Invest 81:945-950

Hudson RR, Kaplan NL (1985) Statistical properties of the number of recombination events in the history of a sample of DNA sequences. Genetics 111:147-164

Kamatani N, Takeuchi F, Nishida Y, Yamanaka H, Hishioka K, Tatara K, Fujimori S, Kaneko K, Akaoka I, Tofuku Y (1985) Severe impairment in adenine metabolism with a partial deficiency of adenine phosphoribosyltransferase. Metabolism 34:164-168
Kamatani N, Kuroshima S, Terai C, Hidaka Y, Pallela TD, Nishioka K (1989) Detection of an amino acid substitution in the mutant enzyme for a special type of adenine phosphoribosyltransferase (APRT) deficiency by sequence-specific protein cleavage. Am J Hum Genet 45:325-331

Kamatani N, Kuroshima S, Hakoda M, Pallela TD, Hidaka Y (1990) Crossovers within a short DNA sequence indicate a long evolutionary history of the APRT*J mutation. Hum Genet 85:600-604

Kamatani N, Hakoda M, Otsuka S, Yoshikawa H, Kashiwazaki S (1992) Only three mutations account for almost all defective alleles causing adenine phosphoribosyltransferase deficiency in Japanese patients. J Clin Invest 90:130-135

Kamatani N, Terai C, Kim SY, Chen CL, Yamanaka H, Hakoda M, Totokawa S, Kashiwazaki S (1996) The origin of the most common mutation of adenine phosphoribosyltransferase among Japanese goes back to a prehistoric era. Hum Genet 98:596-600

Kelley WN, Levy RI, Rosenbloom FM, Henderson JF, Seegmiller JE (1968) Adenine phosphoribosyltransferase deficiency: a previously undescribed genetic defect in man. J Clin Invest 47:2281-2289

Laird N (1993) The EM algorithm. In: Rao CR (ed) Computational statistics. Elsevier, New York, pp 509-520

Lange K (2002) Mathematical and statistical methods for genetic analysis, 2nd edn. Springer, New York Berlin Heidelberg

Laxdal T, Jonasson TA (1988) Adenine phosphoribosyltransferase deficiency in Iceland. Acta Med Scand 224:621-626

Liu JS, Sabatti C, Teng J, Keats BJB, Risch N (2001) Bayesian analysis of haplotypes for linkage disequilibrium mapping. Genome Res 11:1716-1724

Metropolis N, Rosenbluth A, Rosenbluth M, Teller A, Teller E (1953) Equations of state calculations by fast computing machines. J Chem Phys 21:1087-1092

Mimori A, Hidaka Y, Wu VC, Tarle SA, Kamatani N, Kelley WN, Pallela TD (1991) A mutant allele common to the typeI adenine phosphoribosyltransferase deficiency in Japanese subjects. Am J Hum Genet 48:103-107

Mohlke KL, Lange EM, Valle TT, Ghosh S, Magnuson VL, Silander K, Watanabe RM, Chines PS, Bergman RN, Tuomilehto J, Collins FS, Boehnke M (2001) Linkage disequilibrium between microsatellite markers extends beyond $1 \mathrm{cM}$ on chromosome 20 in Finns. Genome Res 11:1221-1226

Sahota AS, Tischfield JA, Kamatani N, Simmonds HA (1995) Adenine phosphoribosyltransferase deficiency and 2,8-dihydroxyadenine lithiasis. In: Scriver CR, Beaudet AL, Sly WS, Valle D, Childs B, Kinzler KW, Voglestein B (eds) The metabolic \& molecular bases of inherited disease. McGraw-hill, New York, pp 2571-2584

Taniguchi A, Hakoda M, Yamanaka H, Terai C, Hikiji K, Kawaguchi R, Konishi N, Kashiwazaki S, Kamatani N (1998) A germline mutation abolishing the original stop codon of the human adenine phosphoribosyltransferase (APRT) gene leads to complete loss of the enzyme protein. Hum Genet 102:197202 